\title{
Research on the Realization of Passive TDOA in PCM/FM System
}

\author{
DAI Tao XIE Nan FENG Lei WANG Yuan Dong \\ Institute of Electronic Engineering, China Academy Engineering Physics, \\ Mian yang, Sichuan, China,daitao8484@163.com
}

\begin{abstract}
:
A kind of PCM/FM telemetry system which can realize the passive time difference of arrival (TDOA) position is introduced in this paper. The positioning principle is hyperbolic algorithm based on least squares method, and the method of system time-measurement is a timing synchronization algorithm based on data transformation tracking loop (DTTL). In this paper, the structure and hardware implementation platform of the positioning system are introduced, and the random error of the system is analyzed. In order to improve the positioning accuracy, the pre filtering method is adopted to reduce the random error caused by noise. The test results show that the positioning error is 18 meter when the $\mathrm{SNR}$ is $8.5 \mathrm{~dB}$ and the source rate is $2 \mathrm{Mbps}$. Therefore, this system can realize the precise positioning of the target signal in PCM/FM system.
\end{abstract}

Key words: TDOA, timing synchronization, cubic interpolation, random error

\section{Introduction}

The passive location techniques estimate the geometric position of the target by detecting the characteristic parameters (such as the electric field intensity, propagation time or time difference, incidence angle, etc.) of the signal which transmitted between the source and multiple receiving stations. The time difference of arrival (TDOA) position technology has the advantages of high precision, high stability and low complexity of equipment, and it has become an important method in the field of reentry telemetry. The PCM/FM has been mainly used in the reentry telemetry transmission system. Therefore, it is necessary to study the multi station passive time difference location of PCM/FM system.

\section{The Positioning Principle}

TDOA positioning, also known as the hyperbolic positioning, by measuring the time difference between the radio signal to reach the different measurement stations to locate the source of the signal. A hyperbolic surface can be obtained by a time difference, when determining the position of the radiation source in the three-dimensional space, three hyperbolic surfaces are required. This will need four or more stations to measure the arrival time of the signal for the source positioning [1]. The sketch map of multi station time difference location system is shown in Fig. 1.

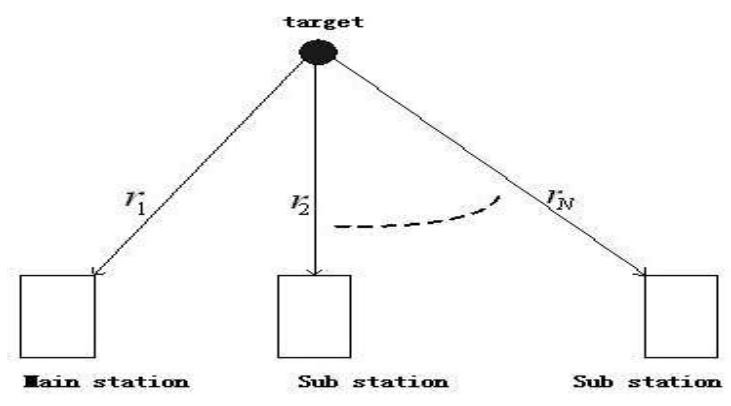

Fig. 1. Sketch map of TDOA

Set the position of the measuring station is

$\left[\begin{array}{lll}x_{i} & y_{i} & z_{i}\end{array}\right]^{\top}(i=1,2, \ldots, N)$, where $i=1$ means the main station, and the rest are sub stations. Set the position of the target is $\left[x_{T} y_{T} z_{T}\right]^{\top}$. The distance between the target and the stations are $r_{i}$, and the distance difference of the target to the main station and the sub stations is $r_{1 i}$.

$$
\left\{\begin{array}{l}
r_{1}^{2}=\left(x_{T}-x_{1}\right)^{2}+\left(y_{T}-y_{1}\right)^{2}+\left(z_{T}-z_{1}\right)^{2} \\
r_{i}^{2}=\left(x_{T}-x_{i}\right)^{2}+\left(y_{T}-y_{i}\right)^{2}+\left(z_{T}-z_{i}\right)^{2} \\
r_{1 \mathrm{i}}=r_{1}-r_{i}=c t_{1 i}
\end{array}\right.
$$

In (1), $t_{1 i}$ is the target signal arrival time difference of the main station and sub stations, $\mathrm{C}$ is the signal transmission speed.

According to the error transmission principle, differential on the third equation of (1), the 
estimation error of target location can be obtained as (2).

$$
d X=\left(C^{T} C\right)^{-1} C^{T}\left[d \Delta R-d X_{s}\right]
$$

where,

$$
\begin{aligned}
c_{i x} & =\frac{x_{T}-x_{i}}{r_{i}}, c_{i y}=\frac{y_{T}-y_{i}}{r_{i}}, c_{i z}=\frac{z_{T}-z_{i}}{r_{i}} \\
k_{i} & =c_{i x} d x_{i}+c_{i y} d y_{i}+c_{i z} d z_{i} \\
C & =\left[\begin{array}{lll}
c_{2 x}-c_{1 x} & c_{2 y}-c_{1 y} & c_{2 z}-c_{1 z} \\
c_{3 x}-c_{1 x} & c_{3 y}-c_{1 y} & c_{3 z}-c_{1 z} \\
c_{4 x}-c_{1 x} & c_{4 y}-c_{1 y} & c_{4 z}-c_{1 z}
\end{array}\right]
\end{aligned}
$$

It represents the relationship between target location and station location;

$$
d \Delta R=\left[d\left(\Delta r_{12}\right), d\left(\Delta r_{13}\right), d\left(\Delta r_{14}\right)\right]
$$

It represents the error introduced by the time difference measurement of each station;

$$
d X_{s}=\left[\Delta k_{12}, \Delta k_{13}, \Delta k_{14}\right]
$$

Where $\Delta k_{1 i}=k_{1}-k_{i}$. It represents the error introduced by site measurement of each station; $\mathrm{dX}$ is the measurement error of target location.

So that, the calculation of target position depends on the measurement of the station location and the target signal arrival time difference to each station. Site measurement can be completed through the self localization by each station. Therefore, the basis of TDOA is the accurately record of the arrival time of the signal, the symbol clock recovery of PCM/FM signal is completed by the timing synchronization, so the timing synchronization is the key point of the system.

\section{Timing Scheme}

In $\mathrm{PCM} / \mathrm{FM}$ all digital receiver, the timing estimation is usually performed after the completion of the frequency discrimination. But due to the threshold effect of FM demodulation, the performance of PCM code stream can be deteriorated sharply when the SNR of input signal is lower than the threshold value. Therefore, in this paper, the timing synchronization is directly carried out after DDC, so as to avoid the influence of the threshold effect. The system structure diagram is as follows.

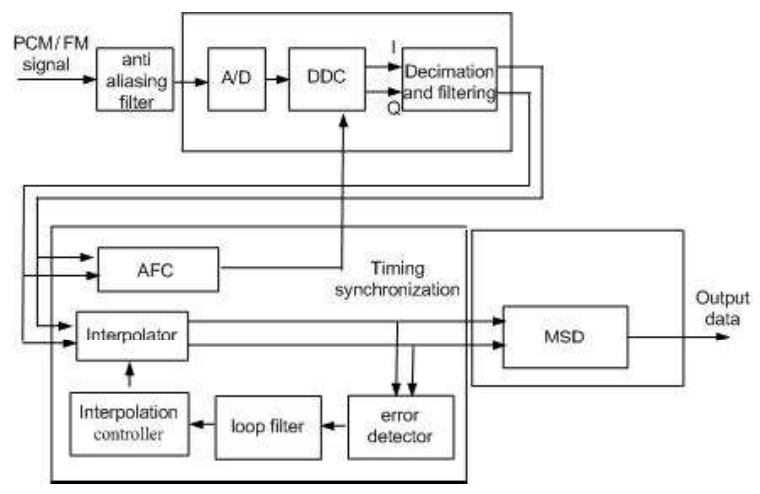

Fig. 2. Elements of all digital receiver.

Because the timing synchronization is carried out prior to the carrier synchronization, we choose the data conversion tracking loop (DTTL) algorithm which is not sensitive to the carrier phase [2].

In order to facilitate the realization of the subsequent signal processing, the $\mathrm{I} / \mathrm{Q}$ data is decimated and filtered to reduce the signal rate. The I/Q signals are performed the same operation to detect the timing error. The process is divided into two branches, each branch has integrator, the integration period is equal to the symbol width, but the two branches staggered half symbol width. The up Branch is used to detect the data conversion and direction, the down branch is used to determine the size of the timing error, multiplying the output of the two branches. Finally, the calculation results of I/Q signals are summed up to yield the final timing error.

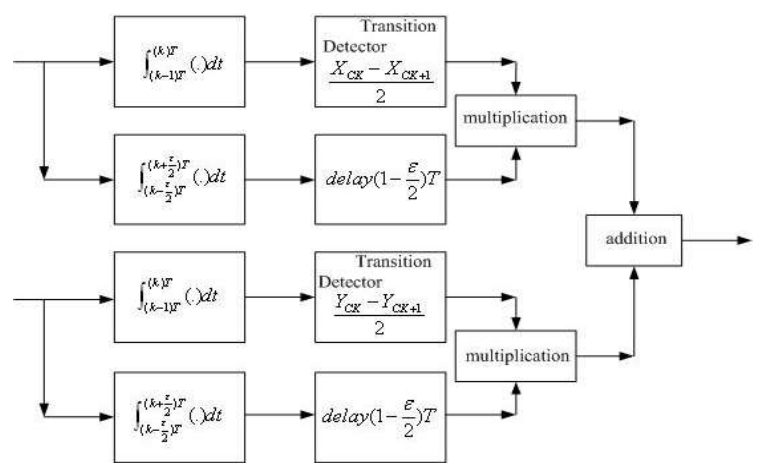

Fig. 3. Block diagram of DTTL.

The obtained error signal pass through the loop filter, and the two-order filter is usually adopted. It also consists of two branches: proportional and integral branches, according to the control theory, we can know that the proportion of branch can track the phase error, but cannot track the frequency error. The coefficients of the two branches determine the bandwidth of the loop and the damping coefficient, which affect the acquisition ability and convergence speed of the timing error, so they are important parameters in the debugging process. 
Due to the sampling clock of the system is independent; the ideal sampling point cannot be got directly. It needs to be calculated by interpolation [3]. The filtered timing error is fed into the interpolation controller to obtain the interpolation point and interpolation interval, and the interpolator completes the calculation to get the ideal sampling point.

It applies polynomial interpolation to correct timing error in the system. Polynomial interpolation can be regarded as low pass filtering, the frequency response of the request is: in the frequency range $0 \sim 1 /\left(2 \mathrm{~T}_{\mathrm{s}}\right)$ with a flat response and linear phase, and the high frequency component of the signal can be suppressed as much as possible. Usually there are three kind of interpolator: linear, parabolic and cubic interpolation. Because of the efficient farrow structure and better interpolation performance, the choice is cubic interpolation; the cost is the increase of computation amount.

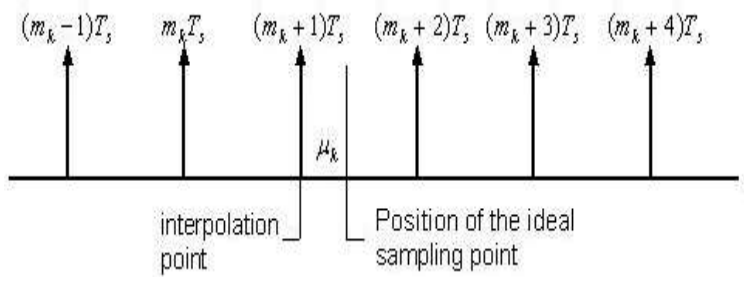

Fig. 4. Timing relation of interpolation

In Fig. 4, $\left(m_{k}+n\right) T_{s}(n=-1, \ldots, 4)$ are the sample times of the digital sequence that is send into the interpolation filter. The sample period is $T_{S}$, $\left(m_{k}+1\right) T_{s}$ is the interpolation point, which is selected by the enable signal that output from the interpolation controller. $\mu_{\mathrm{k}}$ is the interpolation interval, which is the timing error, also output from the controller. It shows that $\left(m_{k}+1\right) T_{s}$ and $\mu_{k}$ reflect the actual position of the ideal sampling point, which is the real arrival time of the signal.

When recording the arrival time of the signal, it is necessary to record $\left(m_{k}+1\right) T_{s}$ and the value of $\mu_{k} .\left(m_{k}+1\right) T_{s}$ can be recorded by high-precision $200 \mathrm{MHz}$ clock and 1PPS signal which output from GPS receiver. $\mu_{\mathrm{k}}$ is used as a correction value to compensate for $\left(m_{k}+1\right) T_{s}$ to ensure the accuracy of the measurement. The structure of time measurement is shown below in Fig. 5 .

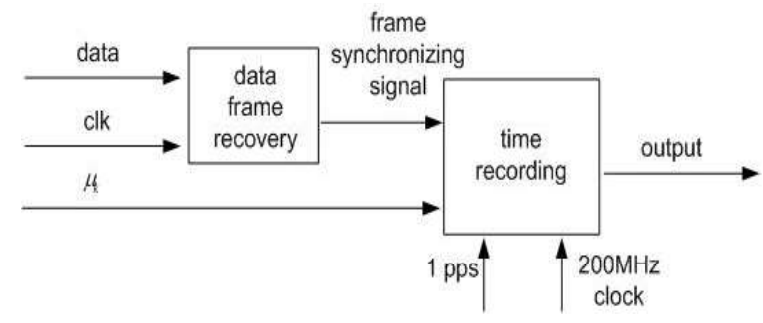

Fig. 5. Structure of time measurement

\section{Error analysis and improvement}

Compare with the site measurement error of each station, the time measurement error $\varepsilon$ is the main measurement error in multi station passive location.

$$
\varepsilon=\sqrt{2 \varepsilon_{c}^{2}+2 \varepsilon_{q}^{2}+\varepsilon_{s}^{2}+\varepsilon_{d}^{2}+\varepsilon_{f}^{2}}
$$

In (8), $\quad \varepsilon_{c}$ is the signal transmission error, it caused by atmospheric delay, when the altitude of the target is low, it is mainly troposphere delay. In engineering applications, it is generally thought to be $5 \mathrm{~ns} ; \varepsilon_{\mathrm{q}}$ is measurement station clock error, it caused by the local clock oscillator inaccuracy and instability, it is considered to be $5 \mathrm{~ns}$ In this system; $\varepsilon_{\mathrm{s}}$ is synchronization error between measurement stations, when using GPS Common-view approach, it generated by the GPS receiver of each station, and it is usually about $10 \mathrm{~ns} ; \varepsilon_{\mathrm{f}}$ is doppler error, it produced by High speed movement of the target, it is usually about $15 \mathrm{~ns}$; $\varepsilon_{d}$ is random error which reflects the timing error jitter[1].

$\varepsilon_{\mathrm{d}}$ is mainly affected by noise and timing loop bandwidth. If only the loop filter is used to reduce the timing error jitter, the loop bandwidth will be reduced, thereby increasing the acquisition time and making the loop stability worse. The noise includes signal noise and timing synchronization self-noise, so the selfnoise can be reduced by using the pre filtering method, and then the timing jitter is reduced. Its structure is shown in Fig. 6.

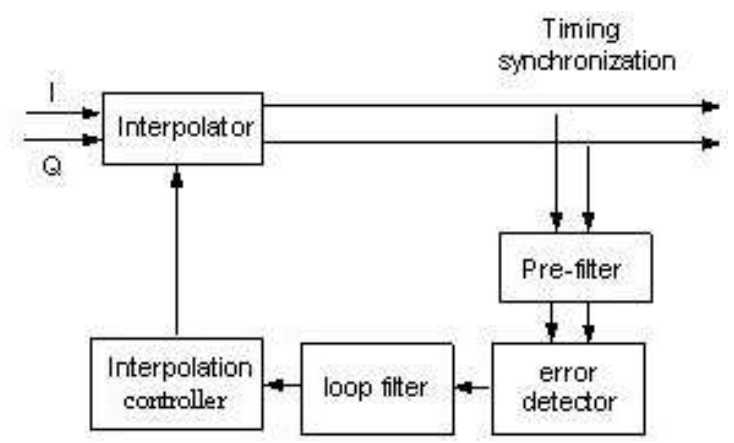

Fig. 6 Elements of timing synchronization with pre-filter

When the even symmetrical bandpass signal which frequency spectrum range in $(1 / 4 \mathrm{~T}, 3 / 4 \mathrm{~T})$ and take $1 / 2 T$ as the center reaches the steady state, the timing jitter is approximately 0 [4]. Therefore, the pre filter should meet the requirements of the spectral characteristics of the signal. Its amplitude frequency characteristic is expressed in (9). 


$$
H_{\mathrm{o}}(f)=\left\{\begin{array}{l}
G\left(f-\frac{1}{T}\right), 0 \leq f<\frac{1}{T} \\
G\left(f+\frac{1}{T}\right),-\frac{1}{T} \leq f<0
\end{array}\right.
$$

Where $G(f)$ is the spectral characteristic expression of the shape filter. So the pre-filter is a symbol rate of the shaped filter in the frequency domain. After adding this filter, the synchronization performance is significantly improved, and the results are as follows.

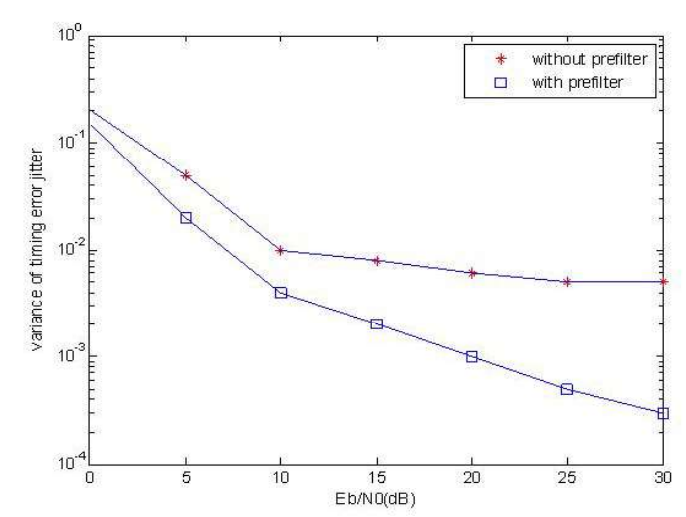

Fig. 7 Restult of matlab simulation

After adding the pre-filter, the timing error jitter $\varepsilon_{d}$ is about $1 / 120$ of the symbol period. When the symbol rate is $2 \mathrm{Mbps}, \varepsilon_{\mathrm{d}}$ is about $4 \mathrm{~ns}$, so the time measurement error $\varepsilon$ is $21 \mathrm{~ns}$. The position error is $6.3 \mathrm{~m}$ introduced by time measurement.

\section{Testing results}

Block diagram of the system testing is shown in Fig. 8, signal source generates radio frequency signal transmitted by antenna, A, B, C, D four receivers receive the signal at the same time. By comparing the time scale information in the demodulation data, and combined with the station's own coordinates, positioning the coordinate of the signal source.

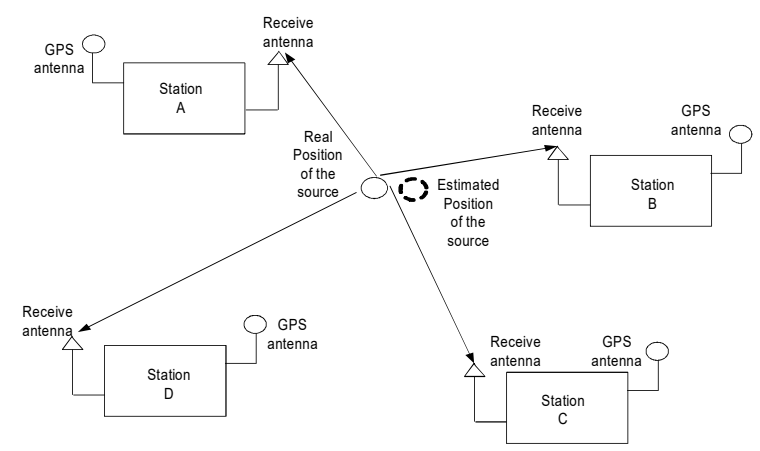

Fig. 8 Sketch map of system test

Where the rate of the test signal is $2 \mathrm{Mbps}$, signal to noise ratio is $8.5 \mathrm{~dB}$, and the distance between the calculated location of the signal source and the actual location is $18 \mathrm{~m}$. So it realize the accurate location of the target.

\section{Conclusions}

This paper introduces the basic principle of multi station TDOA location on PCM/FM signal. According to the principle of positioning, using digital transformation tracking loop estimate timing error, coupled with the time measurement module to complete high precision calibration records. The testing results show that the system can achieve precise positioning of PCM/FM signal source.

\section{References}

[1] Xie Nan. Precision Analysis of Time Difference Locating Technology Base on Multi-ground Stations. Information and Electronic Engineering,2003,1(3):38-42

[2] Marvin K. Simon, Andre Tkacenko. Noncoherent Data Transiton Tracking Loops for Symbol Synchronization in Digital Communication Receivers. IEEE Trans. Communications, 2006,54(5):889-899

[3] GARDNER F M. Interpolation in digital modems - part I: fundamentals. IEEE Trans.Communications, 1993,41(3):501508.

[4] Franks L E, Bubrouski J P. Statistical properties of timing jitter in a PAM timing recovery Scheme. IEEE Trans.

Communications, 1974,22(7):913-920 